АССОЦИАЦИИ ПОЛОВЫХ ГОРМОНОВ С КОМПОНЕНТАМИ ИНСУЛИН-ГЛЮКОЗНОГО ГОМЕОСТАЗА

( ) О.В Цыганкова ${ }^{1,2 *}$, А.Р. Бадин' ${ }^{1}$, З.Г. Бондарева' ${ }^{1}$ Н.Г. Ложкина' , Д.Ю. Платонов

'ФГБОУ ВО Новосибирский государственный медицинский университет, Новосибирск, Россия ${ }^{2}$ НИИ терапии и профилактической медицины, филиал ФГБНУ «Федеральный исследовательский центр Институт цитологии и генетики Сибирского отделения Российской академии наук», Новосибирск, Россия ${ }^{3}$ ФГБОУ ВО Тверской государственный медицинский университет, Тверь, Россия

В данном литературном обзоре предпринята попытка анализа взаимосвязей основных половых гормонов с процессами развития и прогрессирования инсулинорезистентности как фундаментального патогенетического компонента инсулин-глюкозного гомеостаза. При оценке половых стероидов использовался комплексный подход - подробно описаны ассоциации как андрогенов, так и эстрогенов у лиц мужского и женского пола, большое внимание уделено нарушению секреции и эффективности основных адипоцитокинов - лептина и адипонектина в цепи взаимодействий половые гормоны - инсулин - глюкоза. В завершение обзора представлены новые данные об экспрессии натрий-зависимых котранспортеров глюкозы (SGLT) и глюкозных транспортеров (GLUT) у животных в зависимости от пола.

КЛЮЧЕВЫЕ СЛОВА: обзор, инсулинорезистентность, пол, эстрогены, андрогены, лептин, адипонектин, натрий-глюкозные котранспортеры.

\title{
ASSOCIATIONS OF SEX HORMONES WITH COMPONENTS OF INSULIN-GLUCOSE HOMEOSTASIS
}

\author{
(c) Oksana V. Tsygankova1,2* , Artur R. Badin' ', Zoya G. Bondareva', Natalya G. Lozhkina', Dmitrii Y. Platonov ${ }^{3}$
}

${ }^{1}$ Novosibirsk State Medical University, Novosibirsk, Russia

${ }^{2}$ Research institute of therapy and preventive medicine" branch of Federal Research Institute of a Cytology and Genetics, Novosibirsk, Russia

${ }^{3}$ Tver State Medical University, Tver, Russia

In this literature review, an attempt is made to analyze the interrelationships of the main sex hormones with the processes of development and progression of insulin resistance as a fundamental pathogenetic component of insulin-glucose homeostasis. In the evaluation of sex steroids, a complex approach was used - the associations of both androgens and estrogens in males and females are described in detail, a great deal of attention is paid to the violation of the secretion and effectiveness of the main adipocytokines - leptin and adiponectin in the sex hormone-insulin-glucose interaction chain. At the end of the review, new data on the expression of sodium-dependent glucose cotransporter (SGLT) and glucose transporters (GLUT) in animals, depending on sex, are presented.

KEYWORDS: review, insulin resistance, sex, estrogens, androgens, leptin, adiponectin, sodium-glucose transport proteins.

\section{ВВЕДЕНИЕ}

Известно, что нарушения углеводного обмена отчетливо демонстрируют гендерную специфику. У женщин они встречаются чаще, имеют более тяжелое течение и неблагоприятный прогноз. Особое внимание в этой связи привлекают специфичные для пола аспекты патогенетических изменений в системе ожирение-инсулин-глюкоза, обусловленные влиянием половых гормонов. Долгое время общепринятой являлась позиция, согласно которой эстрогены у женщин обладают протективным действием, а андрогены - негативным [1]. С другой стороны, накапливаются данные, что эстрогены как у женщин, так и у мужчин могут оказывать не только положительное, но и отрицательное влияние на метаболические параметры [1].

Негативная роль высоких концентраций эндогенных андрогенов у женщин как модуляторов течения нарушений углеводного обмена до сегодняшнего дня окончательно не определена и в основном ограничивается изучением когорты пациенток с синдромом поликистозных яичников. Данные о положительной ассоциации дефицита тестостерона у мужчин как патогенетического фактора висцерального ожирения, дисбаланса проатерогенных цитокинов, лептинорезистентности и гипергликемии отмечаются в подавляющем большинстве публикаций, однако научные дебаты обусловлены различным пониманием причинно-следственных изменений уровней андрогенов, эстрогенов и компонентов инсулин-глюкозного гомеостаза $[2,3,4]$. Таким образом, несмотря на большой исследовательский материал, посвященный роли половых гормонов у пациентов с изменениями метаболизма инсулина, в настоящее время сохраняется много неизученных вопросов, неопределенных результатов, требующих детализации. 


\section{МУЖСКИЕ ПОЛОВЫЕ СТЕРОИДЫ И ИНСУЛИНОРЕЗИСТЕНТНОСТЬ}

Инсулин и андрогены находятся в сложных взаимозависимых отношениях: как инсулин регулирует синтез и действие андрогенов, так и андрогены влияют на секрецию инсулина и чувствительность к нему. Классическое массачусетское исследование, в котором на протяжении 10 лет изучались процессы старения мужчин, показало, что у мужчин с низким уровнем тестостерона (Т) повышается риск развития инсулинорезистентности (ИР) и сахарного диабета 2 типа (СД2). Позже эти данные были неоднократно подтверждены другими авторами $[5,6]$. Увеличивающееся количество наблюдений о протективном влиянии эндогенных андрогенов на чувствительность к инсулину у мужчин некоторые исследователи объясняют их трансформацией в эстрогены, однако D. Liu и соавт. (2008) показали, что эффект дегидроэпиандростерона сохранялся в присутствии ингибиторов эстрогеновых рецепторов [2]. M. Bekaert и соавт. (2015) также считают, что влияние Т - самостоятельный процесс и не зависит от активности ароматазы и баланса эстрогенов [3].

Существует и обратная направленность вектора «андрогены-ИР»: ожирение, метаболический синдром, СД2 предрасполагают к развитию андродефицита [4]. Сувеличением массы адипоцитов под воздействием ароматазы концентрация циркулирующих эстрогенов возрастает, что приводит к подавлению выработки лютеинизирующего гормона и, следовательно, Т. Висцеральное ожирение также ассоциировано с гиперпродукцией целого ряда гормонов (лептин, цитокины, грелин, инсулин), которые ингибируют ось гипоталамус-гипофиз-яички на разных уровнях и, соответственно, ведут к гипоандрогении [7]. Формирование ИР в условиях недостаточной концентрации андрогенов реализуется через несколько механизмов:

- уменьшение экспрессии инсулиновых рецепторов;

- уменьшение экспрессии субстратов инсулиновых рецепторов, а также их фосфорилирования;

- уменьшение активности ключевых ферментов, открывающих пути утилизации глюкозы через пентозофосфатный путь и цикл трикарбоновых кислот;

- недостаточная активность процессов окислительного фосфорилирования, липидного окисления с внутриклеточной аккумуляцией триглицеридов в скелетных мышцах [8].

При сравнении мужчин с наличием андродефицита и без него в проекте TELECOM обнаружено, что первые имели более высокие показатели триглицеридов, холестерина липопротеидов низкой плотности, общего холестерина, индекса массы тела, соотношения окружность талии/окружность бедер, систолического артериального давления, уровня глюкозы и инсулина натощак и через 2 ч после еды и более низкие уровни а-холестерина. При применении метода мультивариантного анализа после поправки с учетом индекса массы тела и соотношения талия/бедро только уровни инсулина и триглицеридов оставались статистически значимо различными между группами, что свидетельствует о возможности самостоятельного влияния Т на обмен инсулина, независимо от других метаболических изменений [9]. Эти дан- ные в последующем были подтверждены и в различных возрастных группах: для мужчин среднего и пожилого возраста [10].

В этой связи с практических позиций высоковероятно позитивное влияние заместительной терапии Т на чувствительность к инсулину. Действительно, в одной из недавних работ сравнивали выраженность ИР, которую оценивали по скорости внутривенной инфузии глюкозы во время проведения эугликемического гиперинсулинемического клэмпа, в группах мужчин с гипогонадотропным гипогонадизмом и без него. У мужчин с гипогонадизмом скорость инфузии была меньше на $36 \%$; здесь же было отмечено снижение экспрессии инсулиновых рецепторов, субстрата инсулиновых рецепторов и белков-транспортеров GLUT4. При лечении этих пациентов внутримышечными препаратами Т обнаружили увеличение экспрессии указанных выше рецепторов и белков-переносчиков наряду с улучшением чувствительности к инсулину (повышение скорости инфузии глюкозы на 32\%) [11].

В противовес разделяемой большинством исследователей точке зрения о благоприятном влиянии андрогенов у мужчин на сенситивность к инсулину и углеводный обмен, E.J. Gianatti и соавт. (2014) опубликовали несколько иные результаты: по их данным, терапия Т не оказывала благоприятного влияния ни на ИР, оценивавшуюся по индексу НОМА1, ни на гликемический контроль [12]. Проведенный мета-анализ семи рандомизированных контролируемых исследований, в пяти из которых использовался индекс НОМА1, подтвердил теорию снижения ИР на фоне лечения Т; впрочем, две работы с использованием НОМА2 (модифицированного индекса ИР) говорят об отсутствии какого-либо эффекта [13]. Таким образом, влияние андрогенов на ИР все еще не совсем понятно. Несмотря на то, что дизайн исследований схож, результаты их довольно противоречивы. Одним из объяснений сложившейся дихотомии могут являться разнонаправленные эффекты эндо- и экзогенных гормонов, а также различных видов и доз препаратов андрогенов.

У женщин, в отличие от мужчин, вопрос о влиянии андрогенов на процессы ИР практически не изучен. Большинство таких работ проведено на пациентках с синдромом поликистозных яичников (СПКЯ). Это заболевание является типичным примером гиперандрогенного состояния и встречается в популяции в 7-11\% случаев среди женщин фертильного возраста. В течение 20 лет наблюдения около 20\% таких пациенток заболевают СД2 [14]. Однако у женщин с СПКя, помимо гиперандрогении, имеется ряд других факторов, способствующих развитию и прогрессированию ИР, - ожирение, дислипидемия, артериальная гипертония, нарушение фибринолиза. Наряду с этим, фенотипическая изменчивость этого синдрома определяет весьма гетерогенный профиль сочетания продиабетогенных состояний у каждого конкретного индивидуума $[14,15]$.

Определить точку отсчета формирования порочного круга «ИР-гиперандрогения» достаточно проблематично. Согласно современным воззрениям, первоосновой синдрома стромального гипертекоза яичников является ИР [16], и высокий уровень андрогенов является лишь одним из ее проявлений [17]. Инсулин и лютеинизирующий гормон выступают как синергисты, 
стимулируя синтез андрогенов в тека-клетках через активацию цитохрома P450C17a. Описаны и экстраовариальные механизмы развития гиперандрогении при гиперинсулинемии:

- стимуляция активности P450С 17а в надпочечниках;

- подавление продукции печенью белка, связывающего половые гормоны, что приводит к увеличению концентрации свободного Т;

- стимуляция выброса ЛГ в гипофизе [18].

Ряд исследовательских работ различной давности, напротив, склоняется к первичности гиперандрогении. В недавнем исследовании самок мышей после овариоэктомии лечили 5-дигидроэпиандростероном и наблюдали снижение экспрессии GLUT4, повышение уровней глюкозы крови и степени ИР [19]. Другим свидетельством в пользу первостепенности гиперандрогении является снижение ИР под действием антиандрогенной терапии спиронолактоном, флутамидом, бусерелином [20]. Важно подчеркнуть, что в последней работе использовалась оценка ИР с помощью клэмпа - золотого стандарта ее диагностики.

Современные исследования, изучающие связь между уровнями Т и ИР у женщин без СПКЯ, малочисленны. В частности, обнаружено, что у беременных наблюдается положительная корреляция между повышенным уровнем Т и ИР [21]. Гиперандрогения ассоциирована с риском развития метаболического синдрома и, следовательно, ИР у пре- и постменопаузальных женщин [22]. В нашей работе уженщин с ишемической болезнью сердца (ИБС) гиперандрогения была ассоциирована с ранним развитием ИР (оценка проводилась по уровню базальной инсулинемии и индекса НОМА1) уже в возрасте 35-55 лет. В возрастной категории 55-65 лет у пациенток с концентрацией $\mathrm{T} \geq 3$ нмоль/л, в отличие от сверстниц с нормоандрогенией, зафиксировано также увеличение глюкозы плазмы, медиана которой $(7,2$ (5,5; 8,8) ммоль/л) превышала диагностические для СД2 значения, иллюстрируя содружественное негативное влияние возраста и высокого уровня андрогена на уровень гликемии. Полученные результаты были достоверны в парциальном корреляционном анализе с контролем роста и массы тела [1].

Подводя некий промежуточный итог анализа данных, необходимо констатировать сохраняющуюся необходимость дальнейшего изучения взаимоотношений андрогенов с инсулинорезистентным кластером у женщин, не страдающих СПКЯ. Однако пациентки с гиперандрогенией 56-65 лет, имеющие ИБС, уже сегодня нуждаются в регулярном мониторинге гликемии и наблюдении с «диабетической настороженностью».

\section{ЖЕНСКИЕ ПОЛОВЫЕ ГОРМОНЫ И ИНСУЛИНОРЕЗИСТЕНТНОСТЬ}

Установлен целый ряд механизмов, связывающих эстрадиол с повышенной чувствительностью к инсулину уженщин. В частности, 17-бета-этинил-эстрадиол предотвращает аккумуляцию жира посредством супрессии липогенеза, в том числе в печени, и активации липолиза; в скелетной мускулатуре эстрадиол усиливает утилизацию глюкозы, модулируя экспрессию транспортеров глюкозы GLUT4 [23]. Описаны прямые протекторные эффекты эстрогенов в отношении поджелудочной железы, их способность снижать чувствительность к глюкагону и выраженность его секреции, что, в свою очередь, уменьшает глюкагон-индуцированную гипергликемию [23, 24].

Представляется интересной одна из последних работ, выполненная на животных моделях, где показано, что у самок новозеландских мышей с ожирением эстрогены защищают $\beta$-клеточный аппарат поджелудочной железы от разрушения, снижая его чувствительность к глюколипотоксическим состояниям [24]. Авторы предполагают большую выраженность этого эффекта у самок, нежели у самцов, так как у первых связанные с G-белком эстрогеновые рецепторы, предотвращающие апоптоз панкреатических клеток, в значительно большем количестве экспрессируются в островковых клетках поджелудочной железы.

Классическая концепция менопаузы строится на том, что в этом периоде на фоне эстрогеновой недостаточности организм женщины особенно уязвим в отношении прогрессирования атерогенеза, артериальной гипертензии, нарушений липидного, углеводного обмена, ожирения и коагуляционного гомеостаза [25, 26, 27]. С другой стороны, по мнению F. Lizcano, G. Guzmán (2014), первостепенность эстрогенов в развитии метаболических заболеваний во время менопаузы представляется спорной, возможно, ключом к проблеме служит нарушенный обмен андрогенов [28]; аналогичной позиции придерживаются J.S. Brand, Y.T. van der Schouw (2010) [29].

C. Kim и соавт. (2015) при изучении восьми проспективных обсервационных исследований у женщин резюмировали, что у женщин высокий эстрадиол чаще, чем низкий, был ассоциирован с сердечно-сосудистым риском. Авторы считают, что механизм действия половых гормонов, в частности эстрадиола, может быть опосредован геномными, негеномными эффектами, воздействием на общепринятые и другие, менее изученные факторы риска [30]. Предполагается, что действие эстрогенов на сосуды, по крайней мере отчасти, зависит от выраженности атеросклероза. Так, установлено, что экспрессия рецепторов к эстрадиолу значительно снижена в очагах атеротромбоза, в связи с чем прямые эффекты эстрогенов, зависимые от их влияния на рецепторы, в таких артериях будут проявляться в меньшей степени или даже отсутствовать. Возможность разнонаправленного эффекта эстрадиола в зависимости от степени атеросклеротического поражения сосуда разделяют также и отечественные авторы [31]. Учитывая тесную патогенетическую связь эндотелиальной дисфункции и ИР, можно предполагать вклад описанных механизмов и в развитие углеводных нарушений, однако подтверждение или опровержение подобных идей требует дальнейших научных изысканий.

Возвращаясь к вопросам ИР как таковой, необходимо отметить возможность ее раннего развития у плода любого пола на фоне эстрогендефицита у матери, продемонстрированную на приматах [32]. Возможно, эстрогены оказывают благоприятное воздействие на ИР как у мужчин, так и у женщин. В исследовании на крысах терапия эстрогенами снижала показатели HOMA1 у животных обоих полов [33]. Аналогично, в работе A. Inada и соавт. (2016) у самцов мышей, подвергнутых орхиэктомии, терапия эстрогенами усиливала экспрессию GLUT4 
и их транслокацию к клеточной мембране, что снижало уровень гликемии и степень ИР [19].

Интересна эволюция чувствительности к инсулину, наблюдаемая у лиц обоих полов во время пубертата. С наступлением подросткового возраста у юношей чувствительность к инсулину снижается, несмотря на уменьшение процентного количества жировой ткани и увеличения мышечной. У девушек количество жировой ткани, напротив, достоверно увеличивается, однако сенситивность К инсулину значимо не изменяется. Транзиторное снижение чувствительности к инсулину у юношей может быть объяснено развитием относительного дефицита эстрогенов на фоне уменьшения соотношения эстрадиол/Т [34].

Таким образом, в мужском организме не только андрогены, но и эстрогены модулируют клиническое течение и патофизиологические механизмы развития ИР. Концентрация эстрадиола в крови здоровых молодых мужчин, положительно коррелируя с уровнем Т, сравнима с эстрогенной насыщенностью у женщин в ранней фолликулярной фазе менструального цикла и значительно превышает аналогичные уровни у женщин в постменопаузе [1]. Исследование Antonio с участием 3369 европейских мужчин не выявило какой-либо корреляции между базальным уровнем эстрадиола и развитием метаболических нарушений, хотя в многофакторном анализе более низким значениям соотношения эстрадиол/Т соответствовала меньшая вероятность наличия метаболического синдрома. Таким образом, возможно, у взрослых мужчин определяющее значение имеют не абсолютные уровни эстрадиола, а его соотношение с Т [35]. По мнению J. Tökе и соавт. (2014), именно патологическое повышение концентрации Е2 у мужчин приводит к метаболическим нарушениям, включая резистентность к инсулину и СД2, как непосредственно, так и косвенно, за счет снижения концентрации общего и биодоступного Т [36]; аналогичную позицию разделяет G. Williams (2012) [37].

Интересно, что и низкое содержание эстрогенов у мужчин тоже может быть ассоциировано с ИР. Врожденный дефект ароматазы - уникальная модель для изучения роли эстрогенов у мужчин - состояние, характеризующееся изолированным дефицитом эстрогенов на фоне нормального содержания андрогенов, сопряжено с наличием ИР, остеопороза, выраженной дислипидемии и формированием стеатогепатоза [38].

\section{ЛЕПТИН, АДИПОНЕКТИН И ПОЛОВЫЕ ГОРМОНЫ}

Жировая ткань - активный продуцент адипоцитокинов. Некоторые адипокины, например лептин, попадают в системный кровоток (и оказывают системные эффекты), в то время как другие, такие как фактор некроза опухоли-а, интерлейкин-6 и ингибитор активатора плазминогена, локализуются в жировой ткани и функционируют как паракринные или аутокринные регуляторы [39]. Лептин занимает особое место в ряду адипоцитокинов, оказывая регуляторное влияние на различные физиологические процессы, включающие аппетит, массу тела, нейроэндокринные функции, уровень гликемии. Он воздействует на симпатическую и парасимпатическую нервные системы, что приводит к усиленному поглощению глюкозы мышечными клетками, снижению панкреатического синтеза глюкагона и печеночной продукции глюкозы [40]. Благодаря лептину происходит диалог между жировой тканью и гипоталамусом, его называют «голосом жировой ткани» [16]. В последнее время получены данные о возможности секреции лептина плацентой и яичниками, что свидетельствует о его роли в регуляции репродуктивной системы и развитии СПКЯ [41].

Действительно, лептин находится в динамическом балансе с уровнем половых гормонов: он может напрямую влиять на концентрацию эстрадиола, который, в свою очередь, регулирует продукцию лептина адипоцитами по механизму положительной обратной связи [16]. У женщин лептин выступает в качестве индикатора нутриционного статуса, необходимого для зачатия и успешного протекания беременности, активации оси гипоталамус-гипофиз-яичники, что было впервые изучено на моделях мышей с нокаутом соответствующего гена. Экзогенное введение лептина таким животным индуцировало половое созревание, развитие гонад, нормализовало секрецию гонадотропинов и восстанавливало фертильность. В экспериментальных работах введение лептина женщинам с его дефицитом также приводило к увеличению концентраций гонадотропинов и эстрадиола $[42,43]$.

Было высказано предположение, что лептин меньше эволюционировал как гормон сытости и больше как сигнал для репродуктивной системы об адекватном запасе энергии. Уровень лептина повышается на 50\% непосредственно перед началом полового созревания, в дальнейшем наблюдается его стабилизация на протяжении 2 лет. Для девочек характерен стойкий подъем уровня лептина на протяжении всего пубертатного периода с более высокими показателями на поздних стадиях, что соотносится с увеличением эстрогенов [44]. Изменяя секрецию лютеинизирующего гормона, лептин участвует в формировании нарушений менструального цикла при голодании [45]. При адекватном нутритивном статусе лептин усиливает эффекты гонадотропинов, инсулина и соматомедина на стероидогенез в тканях яичника и созревание женских половых клеток - ооцитов [46]. Ожирение сопровождается сложной нейро-гормональной реакцией, нарушающей пути сигнальной трансдукции лептина с развитием лептинорезистентности, ведущей, в свою очередь, к деактивации системы гипоталамус-гипофиз-яичники, нарушению менструального цикла, созревания ооцитов и фолликулов, процессов стероидогенеза в гранулезных и тека-клетках яичников [47].

Примечательно, что у женщин с ожирением уровень лептина выше, чем у мужчин с аналогичным ИМТ [16], что обусловлено специфичным для пола профилем половых стероидов. Актуальность исследования взаимоотношений лептина и андрогенов не вызывает сомнений ввиду неуклонного роста частоты ожирения, ИР и бесплодия в мужской популяции. Вербицкая О.Г. и соавт. (2013) изучали уровень лептина и андрогенов у мальчиков и подростков с ожирением. По их мнению, выявленная на стадии препубертата гиперлептинемия является важным физиологическим регулятором сигнала к началу полового созревания. Повышенный уровень лептина у мальчиков с ожирением в пубертатном возрасте, а также сохраняющаяся повышенная концентрация антимюл- 
лерова гормона, подавляя подъем уровня андрогенов, обусловливает у них развитие пролонгированного пубертата [48].

Гиперлептинемия может привести к глубоким нарушениям репродуктивной системы мужчин с ожирением. Она индуцирует клинический андрогенный дефицит за счет снижения чувствительности андрогеновых рецепторов к Т и блокады синтеза лютеинизирующего гормона в гипофизе, с одной стороны, наряду с усилением ароматизации Т на периферии в эстрадиол под влиянием ароматазы жировой ткани - с другой. Синергичный эффект на выработку андрогенов оказывает активизация в условиях лептинорезистентности окислительного стресса на фоне избытка свободных жирных кислот и триглицеридов в крови [49].

Локальное нарушение выработки лептина у мужчин с отложением жира в мошонке может быть, по мнению некоторых исследователей, важной причиной нарушения сперматогенеза и андродефицита. Они предположили, что скротальный липоматоз ведет к повышению температуры мошонки посредством избыточной жировой изоляции, венозного застоя и высокого расположения яичка в мошонке на фоне ожирения [50].

В 1995-1996 гг. независимыми группами японских и американских ученых был открыт еще один белковый гормон, синтезируемый белыми адипоцитами, - адипонектин, название которого имеет многочисленные синонимы: ACRp30 (adipocyte complement-related protein $30 \mathrm{kDa}$ ), adipoQ и GBP28 (gelatin binding protein $28 \mathrm{kDa}$ ). Адипонектин обладает противовоспалительным и антиатерогенным действием, что объясняет его отрицательную корреляцию с массой жировой ткани [44]. Вместе с тем уровень адипонектина как у здоровых, так и больных СД2 женщин выше, чем у мужчин [51]. С возрастом плазменная концентрация адипонектина у женщин значительно не меняется. С другой стороны, мужчины старше 70 лет имеют значительно более высокие уровни адипонектина, чем те, кто не достиг этого возраста. Эти данные позволяют предположить, что уровни эстрогенов не оказывают влияния на концентрацию адипонектина, а андрогены подавляют его секрецию [52].

Солнцева А.В. и соавт. (2011) детально изучили гендерные различия адипонектина у детей с алиментарным ожирением и обнаружили более высокие уровни у мальчиков в пубертатном возрасте в сравнении с девочками. Пиковые концентрации были отмечены у мальчиков в периоде раннего пубертата со снижением их к завершению полового созревания, что авторы объясняют отрицательным влиянием повышенного уровня тестикулярных андрогенов на продукцию адипоцитокина. У девочек с развитием пубертата выявлено уменьшение показателей адипонектинемии [53].

Известно, что у женщин адипонектин регулирует выработку гормонов и экспрессию генов в соматотрофах и гонадотрофах гипофиза, ингибируя секрецию лютеинизирующего гормона, но не оказывает влияния на концентрации фолликулотропина. Также адипонектин способен подавлять образование Т в яичниках [44]. В недавнем исследовании у взрослых женщин с СПКЯ при изучении в рамках кластерного анализа корреляций 30 адипоцитокинов, ростовых факторов, растворимых молекул адгезии, лизосомальных протеаз с андрогена- ми, лептин и адипонектин продемонстрировали самые сильные независимые связи [54].

Значимая роль дефицита адипонектина для формирования ИР именно в женской, но не в мужской популяции обнаружена G.A. Bonneau и соавт. (2014) [55]. L.D. Høеg и соавт. (2013), напротив, пишут о том, что уровень плазменного адипонектина ассоциирован с фосфорилированием АМФ-активируемой протеинкиназы только у мужчин. Также у женщин имеется более низкая экспрессия AdipoR1 в скелетных мышцах и более низкая концентрация адипонектинчувствительных мышечных волокон 2 типа, детерминирующие меньшую чувствительность женщин к действию адипонектина [56]. Таким образом, можно заключить, что гормоны жировой ткани как компоненты инсулин-глюкозного гомеостаза, разнонаправленно изменяясь, участвуют в выработке и функционировании половых гормонов на различных этапах становления и функционирования репродуктивной системы, вплоть до ее угасания, как прямо, так и опосредованно. Детализация этих взаимоотношений является предметом дальнейшего изучения.

\section{ГЛЮКОЗУРИЯ И ПОЛОВЫЕ ГОРМОНЫ}

В физиологических условиях почки фильтруют до 180 г глюкозы в сутки, при этом практически вся она полностью реабсорбируется с помощью натрий-зависимых котранспортеров глюкозы (sodium-glucose cotransporter - SGLT) в проксимальных извитых канальцах. Выделяют 11 типов таких переносчиков, среди которых первостепенное значение имеют SGLT 2 типа (SGLT2), расположенные в S1-сегменте проксимального извитого канальца и осуществляющие реабсорбцию до 90\% профильтрованной глюкозы. Остальные 10\% глюкозы реабсорбируются SGLT 1 типа (SGLT1) в S2- и S3-сегментах проксимальных извитых канальцев. Молекулы глюкозы, поступившие в эпителиальные клетки почечных канальцев, связываются глюкозными транспортерами (GLUT) и с помощью пассивного транспорта выделяются в кровь [57].

В связи с появлением принципиально нового класса органопротективных и прогнозмодифицирующих сахароснижающих препаратов, механизм действия которых автономен от инсулина и сенситивности тканей к нему, представляет интерес возможность его индивидуального эффекта в зависимости от пола. В исследованиях на мышах было обнаружено, что самки имеют большую концентрацию белков-транспортеров GLUT и экспрессию рецепторов SGLT, чем самцы $[58,59]$. Проведенная овариоэктомия не оказывала никакого влияния на количество натрий-зависимых котранспортеров глюкозы [60]. Кастрация самцов ассоциировалась с увеличением экспрессии SGLT1, а дальнейшая заместительная терапия Т приводила к обратному эффекту. Выявленные половые различия, по мнению авторов, ассоциированы с ингибирующим влиянием андрогенов. Более поздняя работа, где исследовался 2 тип SGLT, в целом подтверждает эти данные: экспрессия транспортера выше у самок мышей и снижается при лечении андрогенами самцов мышей после кастрации. Однако лечение эстрадиолом самок после овариоэктомии все же увеличивало экспрессию SGLT2 [60]. В этой связи теоретически можно предполагать меньшую предрасположенность женщин 
к глюкозурии наряду с большей эффективностью у них ингибиторов SGLT. Несмотря на это, результаты крупных проектов последних лет: EMPA-REG OUTCOME, CVD-REAL, CANVAS свидетельствуют об отсутствии различий в сахароснижающем эффекте на фоне терапии натрий-глюкозными котранспортерами у лиц с СД2 различного пола.

\section{ЗАКЛЮЧЕНИЕ}

Взаимосвязь между ожирением и нарушением фертильности у мужчин и женщин давно доказана, однако изучение уровня андрогенов и эстрогенов сегодня выходит за рамки репродуктивной медицины. В связи с надвигающейся пандемией СД и ожирения представляет интерес изучение дополнительных, «неклассических» факторов риска и аспектов патогенеза этих нозологий, в частности, дисбаланса основных половых гормонов у лиц обоих полов с более глубоким пониманием патогенетических механизмов, обуславливающих гендер- специфические различия в развитии и исходах нарушений углеводного обмена. Уточнение индивидуального профиля риска пациентов с ИР/гипергликемией, включающего андрогены и эстрогены, позволит выявить уязвимые звенья для активного медикаментозного и немедикаментозного воздействия. Планирование и проведение в будущем рандомизированных контролируемых исследований с «твердыми конечными точками» может открыть новые горизонты в использовании половых гормонов или их модуляторов в терапии и профилактике хронических неинфекционных заболеваний.

\section{ДОПОЛНИТЕЛЬНАЯ ИНФОРМАЦИЯ}

Источник финансирования. Поисково-аналитическая работу осуществлена на личные средства авторского коллектива.

Конфликт интересов. Авторы декларируют отсутствие явных и потенциальных конфликтов интересов, связанных с публикацией настоящей статьи.

\section{СПИСОК ЛИТЕРАТУРЫ | REFERENCES}

1. Цыганкова О.В. Этиопатогенетические особенности ишемической болезни сердца в зависимости от уровня половых гормонов, пола и возраста: Дис. ... док. мед. наук. - Новосибирск; 2016. [Tsygankova OV. Etiopatogeneticheskie osobennosti ishemicheskoi bolezni serdtsa $v$ zavisimosti ot urovnya polovykh gormonov, pola i vozrasta. [dissertation] Novosibirsk; 2016. (In Russ).] Доступно по: http://rsmu.ru/fileadmin/rsmu/img/about_rsmu/disser/8/d_ cygankova_ov.pdf. Ссылка активна на 09.04.2017.

2. Liu D, Iruthayanathan M, Homan LL, et al. Dehydroepiandrosterone Stimulates Endothelial Proliferation and Angiogenesis through Extracellular Signal-Regulated Kinase 1/2-Mediated Mechanisms. Endocrinology. 2008;149(3):889-898. doi: 10.1210/en.2007-1125.

3. Bekaert M, Van Nieuwenhove Y, Calders P, et al. Determinants of testosterone levels in human male obesity. Endocrine. 2015;50(1):202211. doi: 10.1007/s12020-015-0563-4.

4. Haring $R$, Ittermann $T$, Völzke $H$, et al. Prevalence, incidence and risk factors of testosterone deficiency in a population-based cohort of men: results from the study of health in Pomerania. The Aging Male. 2010;13(4):247-257. doi: 10.3109/13685538.2010.487553.

5. Stellato RK, Feldman HA, Hamdy O, et al. Testosterone, sex hormonebinding globulin, and the development of type 2 diabetes in middleaged men: prospective results from the Massachusetts male aging study. Diabetes Care. 2000;23(4):490-494. doi: 10.2337/diacare.23.4.490.

6. Høst C, Gormsen LC, Hougaard DM, et al. Acute and Short-term Chronic Testosterone Fluctuation Effects on Glucose Homeostasis, Insulin Sensitivity, and Adiponectin: A Randomized, DoubleBlind, Placebo-Controlled, Crossover Study. J. Clin. Endocr. Metab. 2014;99(6):E1088-E1096. doi: 10.1210/jc.2013-2807.

7. Grossmann M. Testosterone and glucose metabolism in men: current concepts and controversies. J. Endocrinol. 2013;220(3):R37-R55. doi: 10.1530/joe-13-0393.

8. Rao PM, Kelly DM, Jones TH. Testosterone and insulin resistance in the metabolic syndrome and T2DM in men. Nature Reviews Endocrinology. 2013;9(8):479-493. doi: 10.1038/nrendo.2013.122.

9. Simon D, Charles M-A, Nahoul K, et al. Association between Plasma Total Testosterone and Cardiovascular Risk Factors in Healthy Adult Men: The Telecom Study1. J. Clin. Endocr. Metab. 1997;82(2):682-685. doi: 10.1210/jcem.82.2.3766

10. Zhang $\mathrm{N}$, Zhang $\mathrm{H}$, Zhang $\mathrm{X}$, et al. The relationship between endogenous testosterone and lipid profile in middle-aged and elderly Chinese men. European journal of endocrinology/European Federation of Endocrine Societies. 2014;170(4):487-494. doi: 10.1530/eje-13-0802.

11. Dhindsa S, Ghanim H, Batra M, et al. Insulin Resistance and Inflammation in Hypogonadotropic Hypogonadism and Their Reduction After Testosterone Replacement in Men With Type 2 Diabetes. Diabetes Care. 2016;39(1):82-91. doi: 10.2337/dc15-1518.

12. Gianatti EJ, Dupuis P, Hoermann R, et al. Effect of Testosterone Treatment on Glucose Metabolism in Men With Type 2 Diabetes: A
Randomized Controlled Trial. Diabetes Care. 2014;37(8):2098-2107. doi: $10.2337 / \mathrm{dc} 13-2845$.

13. Grossmann M, Hoermann R, Wittert G, Yeap BB. Effects of testosterone treatment on glucose metabolism and symptoms in men with type 2 diabetes and the metabolic syndrome: a systematic review and meta-analysis of randomized controlled clinical trials. Clin. Endocrinol. (Oxf.). 2015;83(3):344-351. doi: 10.1111/cen.12664.

14. Macut D, Antić IB, Bjekić-Macut J. Cardiovascular risk factors and events in women with androgen excess. J. Endocrinol. Invest. 2014;38(3):295-301. doi: 10.1007/s40618-014-0215-1.

15. Stuckey BGA, Opie N, Cussons AJ, et al. Clustering of metabolic and cardiovascular risk factors in the polycystic ovary syndrome: a principal component analysis. Metabolism. 2014;63(8):1071-1077. doi: 10.1016/j.metabol.2014.05.004.

16. Сметник В.П. Половые гормоны и жировая ткань // Ожирение и метаболизм. - 2007. - Т. 4. - №3. - С. 17-22. [Smetnik VP. Polovye gormony i zhirovaya tkan'. Obesity and metabolism. 2007;4(3):17-22. (In Russ).] doi: 10.14341/2071-8713-5017

17. Гамидов С.И., Иремашвили В.В. Метаболический синдром в урологии. М.: Издательство Инсайт Полиграфик; 2010. [Gamidov SI, Iremashvili WV. Metabolicheskii sindrom v urologii. Moscow: Insait Poligrafik; 2010. (In Russ).]

18. Barber TM, Dimitriadis GK, Andreou A, Franks S. Polycystic ovary syndrome: insight into pathogenesis and a common association with insulin resistance. Clin. Med. (Northfield II.). 2016;16(3):262-266. doi: 10.7861/clinmedicine.16-3-262.

19. Inada A, Fujii NL, Inada O, et al. Effects of $17 \beta$-Estradiol and Androgen on Glucose Metabolism in Skeletal Muscle. Endocrinology. 2016:157(12):4691-4705. doi: 10.1210/en.2016-1261.

20. Moghetti P, Tosi F, Castello $R$, et al. The insulin resistance in women with hyperandrogenism is partially reversed by antiandrogen treatment: evidence that androgens impair insulin action in women. J. Clin. Endocr. Metab. 1996;81(3):952-960. doi: 10.1210/jcem.81.3.8772557.

21. Villarroel C, Salinas A, López P, et al. Pregestational type 2 diabetes and gestational diabetes exhibit different sexual steroid profiles during pregnancy. Gynecol. Endocrinol. 2016;33(3):212-217. doi: 10.1080/09513590.2016.1248933.

22. Valderhaug TG, Hertel JK, Nordstrand N, et al. The association between hyperandrogenemia and the metabolic syndrome in morbidly obese women. Diabetol. Metab. Syndr. 2015;7(1). doi: 10.1186/s13098-015-0040-5.

23. JelenikT, Roden M. How Estrogens Prevent From Lipid-Induced Insulin Resistance. Endocrinology. 2013;154(3):989-992. doi: 10.1210/en.2013-1112

24. Vogel H, Mirhashemi F, Liehl B, et al. Estrogen Deficiency Aggravates Insulin Resistance and Induces $\beta$-Cell Loss and Diabetes in Female New Zealand Obese Mice. Horm. Metab. Res. 2013;45(06):430-435. doi: $10.1055 / s-0032-1331700$ 
25. Dehlendorff C, Andersen KK, Olsen TS. Sex Disparities in Stroke: Women Have More Severe Strokes but Better Survival Than Men Journal of the American Heart Association. 2015;4(7):e001967. doi: 10.1161/jaha.115.001967.

26. Sciacqua A, Perticone M, Tassone EJ, et al. Uric acid is an independent predictor of cardiovascular events in post-menopausal women. Int. J. Cardiol. 2015;197:271-275. doi: 10.1016/j.ijcard.2015.06.069.

27. Smith GN. The Maternal Health Clinic: Improving women's cardiovascular health. Semin. Perinatol. 2015;39(4):316-319. doi: 10.1053/j. semperi.2015.05.012.

28. Lizcano F, Guzmán G. Estrogen Deficiency and the Origin of Obesity during Menopause. BioMed Research International. 2014;2014:1-11. doi: 10.1155/2014/757461

29. Brand JS, van der Schouw YT. Testosterone, SHBG and cardiovascular health in postmenopausal women. Int. J. Impot. Res. 2010;22(2):91104. doi: 10.1038/ijir.2009.64

30. Kim C, Cushman M, Kleindorfer D, et al. A Review of the Relationships between Endogenous Sex Steroids and Incident Ischemic Stroke and Coronary Heart Disease Events. Curr. Cardiol. Rev. 2015;1 1(999):1-1. doi: 10.2174/1573403×11666150107160016.

31. Лебедева А.Ю., Клыков Л.Л., Зайцева В.В. ИБС у молодых женщин: проблемы диагностики и профилактики // Российский кардиологический журнал. - 2011. - №6 - C. 90-97. [Lebedeva AY, Klykov LL, Zaitseva W. Coronary heart disease in young women: problems of diagnostics and prevention. Russian Journal of Cardiology. 2011;(6):90-97. (In Russ.)] doi: 10.15829/1560-4071-2011-6-90-97

32. Maniu A, Aberdeen GW, Lynch TJ, et al. Estrogen deprivation in primate pregnancy leads to insulin resistance in offspring. J. Endocrinol. 2016;230(2):171-183. doi: 10.1530/joe-15-0530.

33. Pratchayasakul W, Chattipakorn N, Chattipakorn SC. Effects of estrogen in preventing neuronal insulin resistance in hippocampus of obese rats are different between genders. Life Sci. 2011;89(1920):702-707. doi: 10.1016/j.lfs.2011.08.011.

34. Moran A, Jacobs DR, Steinberger J, et al. Changes in Insulin Resistance and Cardiovascular Risk During Adolescence: Establishment of Differential Risk in Males and Females. Circulation. 2008;117(18):23612368. doi: $10.1161 /$ circulationaha.107.704569.

35. Antonio L, Wu FCW, O'Neill TW, et al. Associations Between Sex Steroids and the Development of Metabolic Syndrome: A Longitudinal Study in European Men. J. Clin. Endocr. Metab. 2015;100(4):1396-1404. doi: 10.1210/jc.2014-4184

36. Töke J, Czirják G, Bezzegh A, et al. Effects and significance of estradiol in men. Orv. Hetil. 2014;155(23):891-896. doi: 10.1556/oh.2014.29914.

37. Williams G. Aromatase up-regulation, insulin and raised intracellular oestrogens in men, induce adiposity, metabolic syndrome and prostate disease, via aberrant ER-a and GPER signalling. Mol. Cell. Endocrinol. 2012;351(2):269-278. doi: 10.1016/j.mce.2011.12.017.

38. McDermott MT. Endocrine secrets. 6th ed. Philadelphia: Saunders; 2013.

39. Michalakis K, Mintziori G, Kaprara A, et al. The complex interaction between obesity, metabolic syndrome and reproductive axis: A narrative review. Metabolism. 2013;62(4):457-478. doi: 10.1016/j. metabol.2012.08.012.

40. Coppari R, Bjørbæk C. Leptin revisited: its mechanism of action and potential for treating diabetes. Nature Reviews Drug Discovery. 2012;11(9):692-708. doi: 10.1038/nrd3757.

41. Hausman GJ, Barb CR. Adipose Tissue and the Reproductive Axis: Biological Aspects. Endocrine development. 2010;19:31-44. doi: 10.1159/000316895.

42. Donato JJ, Cravo RM, Frazão R, Elias CF. Hypothalamic Sites of Leptin Action Linking Metabolism and Reproduction. Neuroendocrinology. 2011;93(1):9-18. doi: 10.1159/000322472.

43. Горбатенко Н.В., Беженарь В.Ф., Фишман М.Б. Влияние ожирения на развитие нарушения репродуктивной функции у женщин (аналитический обзор литературы) // Ожирение и метаболизм. - 2017. - T.14. - № 1 - C.3-8. [Gorbatenko NV, Bezhenar VF, Fishman MB. Obesity and reproductive health of women. Obesity and metabolism. 2017;14(1):3-8. (In Russ).] doi:10.14341/OMET201713-8

44. Ковалева Ю.В. Гормоны жировой ткани и их роль в формировании гормонального статуса и патогенезе метаболических нарушений у женщин // Артериальная zипертензия. - 2015. - Т.21 - № 4 - C.356-370. [Kovalyova YV. Adipose tissue hormones and their role for female fertility and metabolic disorders. Arterial hypertension. 2015;21(4):356-370. (In Russ).]
45. Santos ED, Pecquery R, Mazancourt Pd, Dieudonné M-N. Adiponectin and Reproduction. Vitam Horm. 2012;90:187-209. doi: 10.1016/b978-0-12-398313-8.00008-7.

46. Michalakis K, Mintziori G, Kaprara A, et al. The complex interaction between obesity, metabolic syndrome and reproductive axis: A narrative review. Metabolism. 2013;62(4):457-478. doi: 10.1016/j. metabol.2012.08.012.

47. Sharma A, Bahadursingh S, Ramsewak S, Teelucksingh S. Medical and surgical interventions to improve outcomes in obese women planning for pregnancy. Best Practice \& Research Clinical Obstetrics \& Gynaecology. 2015;29(4):565-576. doi: 10.1016/j.bpobgyn.2014.12.003.

48. Вербицкая О.Г., Попова В.А., Афонин А.А., и др. Клиникодиагностическое значение определения лептина и андрогенов у мальчиков и подростков с ожирением // Медииинский вестник Юга России. - 2013. - № 2. - C.37-42. [Verbitskaya OG, Popova VA, Afonin AA, et al. Clinicodiagnostic value of determination of leptin and androgenic hormones in boys and teenagers with obesity. Medical Herald of the South of Russia. 2013;(2):37-43. (In Russ).] doi:10.21886/2219-8075-2013-2-37-43

49. Калинченко С.Ю., Тюзиков И.А., Ворслов Л.О., Тишова Ю.А. Ожирение, инсулинорезистентность и репродуктивное здоровье мужчины: патогенетические взаимодействия и современная патогенетическая фармакотерапия // Эффективная фармакотерапия. - 2015. - № 27. - C.66-79. [Kalinchenko SY, Tyuzikov IA, Vorslov LO, Tishova YA. Insulin Resistance and Male Reproductive Health: Pathogenic Interactions and Pathogenetic Pharmacotherapy. Effective Pharmacotherapy. 2015;(27):66-79. (In Russ).]

50. ShaflK A, Olfat S. Scrotal Lipomatosis. Br. J. Urol. 1981;53(1):50-54. doi: 10.1111/j.1464-410X.1981.tb03128.x.

51. Aleidi S, Issa A, Bustanji H, et al. Adiponectin serum levels correlate with insulin resistance in type 2 diabetic patients. Saudi Pharmaceutical Journal. 2015;23(3):250-256. doi: 10.1016/j.jsps.2014.11.011.

52. Adamczak M, Rzepka E, Chudek J, Wiecek A. Ageing and plasma adiponectin concentration in apparently healthy males and females. Clin. Endocrinol. (Oxf.). 2005;62(1):114-118. doi: 10.1111/j.13652265.2004.02182.x.

53. Солнцева А.В., Аксенова Е.А., Сукало А.В., и др. Гендерные различия и генетический полиморфизм адипонектина у детей с алиментарНЫМ ОЖирением // ВЕСЦІ НАЦЫЯНАЛЬНАЙ АКАДЭМІ НАВУК БЕЛАРУСІ. СЕРЫЯ МЕДЫЦЫЫНСКІХ НАВУК. - 2011. - № 2. - C.29-37. [Solntseva AV, Askionova EA, Sukalo AV, et al. Gender changes and genetic polymorphism of adiponectin in children with obesity. Vestsi natsyyanal'nai Akademii Navuk belarusi. Seryya medytsynskikh navuk. 2011;(2):29-37. (In Russ).]

54. Baek K-H, Daan NMP, Koster MPH, et al. Biomarker Profiles in Women with PCOS and PCOS Offspring; A Pilot Study. PLoS One. 2016;11(11):e0165033. doi: 10.1371/journal.pone.0165033.

55. Bonneau GA, Pedrozo WR, Berg G. Adiponectin and waist circumference as predictors of insulin-resistance in women. Diabetes \& Metabolic Syndrome: Clinical Research \& Reviews. 2014;8(1):3-7. doi: 10.1016/j.dsx.2013.10.005

56. Høeg LD, Sjøberg KA, Lundsgaard A-M, et al. Adiponectin concentration is associated with muscle insulin sensitivity, AMPK phosphorylation, and ceramide content in skeletal muscles of men but not women. J. Appl. Physiol. 2013;114(5):592-601. doi: 10.1152/japplphysiol.01046.2012.

57. Мкртумян А.М., Маркова Т.Н., Мищенко Н.К. Влияние ингибиторов натрий-зависимых котранспортеров глюкозы 2 типа на уровень гликированного гемоглобина и массу тела у больных сахарным диабетом 2 типа // ДОКТОР.РУ. - 2016. - № 3. - С.55-58. [Mkrtumyan AM, Markova TN, Mishchenko NK. Effects of Sodium-Glucose Cotransporter 2 Inhibitors on Glycosylated Hemoglobin Levels and Body Weight in Patients with Type 2 Diabetes Mellitus. Doctor.Ru. 2016;(3):55-58. (In Russ).]

58. Nagao K, Yoshida S, Konishi H. Gender differences in the gene expression profiles of glucose transporter GLUT class I and SGLT in mouse tissues. Die Farmazie. 2014 Nov;69(1 1):856-9.

59. Sabolić I, Škarica M, Gorboulev V, et al. Rat renal glucose transporter SGLT1 exhibits zonal distribution and androgen-dependent gender differences. American Journal of Physiology-Renal Physiology. 2006;290(4):F913-F926. doi: 10.1152/ajprenal.00270.2005.

60. Sabolić I, Vrhovac I, Eror DB, et al. Expression of Na+-d-glucose cotransporter SGLT2 in rodents is kidney-specific and exhibits sex and species differences. American Journal of Physiology-Cell Physiology. 2012;302(8):C1174-C1188. doi: 10.1152/ajpcell.00450.2011. 


\section{ИНФОРМАЦИЯ ОБ АВТОРАХ [AUTHORS INFO]}

*Цыганкова Оксана Васильевна, д.м.н., доцент [Oksana V. Tsygankova, ScD, associate professor]; aдpec: Россия, 630091, Новосибирск, Красный пр., д. 52 [address: 52 Krasny Prospect, 630091 Novosibirsk, Russia]; ORCID: http://orcid.org/0000-0003-0207-7063; eLibrary SPIN: 1817-4484; e-mail: oksana_c.nsk@mail.ru.

Бадин Артур Романович, клинический ординатор НГМУ [Artur R. Badin, resident]; ORCID: http://orcid.org/0000-0001-9964-5463; eLibrary SPIN: 7566-0360; e-mail: akherousin@mail.ru.

Бондарева Зоя Геннадьевна, д.м.н., профессор [Zoya G. Bondareva, ScD, professor];

ORCID: http://orcid.org/0000-0002-4725-8565; eLibrary SPIN: 5872-0756; e-mail: zoya.bondareva.2017@mail.ru.

Ложкина Наталья Геннадьевна, д.м.н., доцент [Natalya G. Lozhkina, ScD, associate professor]; ORCID: http://orcid.org/0000-0002-4832-3197; eLibrary SPIN: 5320-7554; e-mail: lozhkina.n@mail.ru.

Платонов Дмитрий Юрьевич, д.м.н., профессор [Dmitrii Y. Platonov, ScD, professor];

ORCID: http://orcid.org/0000-0003-0635-3571; eLibrary SPIN: 6060-2112; e-mail: diplato64@mail.ru.

\section{ЦИТИРОВАТЬ:}

Цыганкова О.В., Бадин А.Р., Бондарева З.Г., Ложкина Н.Г., Платонов Д.Ю. Ассоциации половых гормонов с компонентами инсулин-глюкозного гомеостаза // Ожирение и метаболизм. — 2018. — Т.15. — № 2 - С. 3-10. doi: 10.14341/OMET9482

\section{TO CITE THIS ARTICLE:}

Tsygankova OV, Badin AR, Bondareva ZG, Lozhkina NG, Platonov DY. Associations of sex hormones with components of insulin-glucose homeostasis. Obesity and metabolism. 2018;15(2):3-10. doi: 10.14341/OMET9482 